\title{
A comparison of two sequential geomagnetic polarity transitions (upper Olduvai and lower Jaramillo) from the Southern Hemisphere
}

\author{
B.M. Clement * and D.V. Kent \\ Lamont - Doherty Geological Observatory and Department of Geological Sciences, Columbia University, Palisades, NY IO964 (U.S.A.)
}

(Received August 20, 1984; revision accepted March 27, 1985)

\begin{abstract}
Clement, B.M. and Kent, D.V., 1985. A comparison of two sequential geomagnetic polarity transitions (upper Olduvai and lower Jaramillo) from the Southern Hemisphere. Phys. Earth Planet. Inter., 39: 301-313.

Two normal to reverse ( $\mathrm{N}$ to $\mathrm{R})$ geomagnetic polarity transitions were obtained from a Southern Hemisphere deep-sea sediment core $\left(35.91^{\circ} \mathrm{S}, 59.97^{\circ} \mathrm{E}\right)$. The upper Jaramillo reversal was continuously sampled by taking $0.5 \mathrm{~cm}$ thick samples across $55 \mathrm{~cm}$ of section, and the upper Olduvai reversal was sampled in a similar manner across $60 \mathrm{~cm}$. Both records are characterized by full normal and reverse polarity directions which are in very good agreement with those predicted by axial dipole fields for the core site latitude. Each record also exhibits a zone yielding intermediate directions. Because of a large increase in both the within-level and the between-level scatter in the upper Jaramillo transition zone, it is not considered to represent an accurate record of the geomagnetic field. The upper Olduvai transition, however, appears to be a more coherent record. It is characterized by directions that shallow early in the reversal but then rapidly steepen to nearly vertical, upward directions. The declinations do not change until the inclinations have passed through the vertical. The total directional change occurs within a broad NRM intensity minimum. The virtual geomagnetic pole (VGP) path calculated for this record is not longitudinally constrained but instead exhibits a westward progression through the reversal.

When considered together with the lower Jaramillo transition previously reported from this core, these records constitute a set of sequential Southern Hemisphere transitions. A number of striking similarities exist in the two records such as steep, upward directions and a shallowing which occurs between the near vertical and the full polarity directions. The results of zonal harmonic modeling of these records are consistent with the idea of a standing field which persisted across both the upper Olduvai and lower Jaramillo reversals.
\end{abstract}

\section{Introduction}

The number of paleomagnetic records of polarity transitions which have recently become available (Valet and Laj, 1981; Bogue and Coe, 1982; Williams and Fuller, 1982; Valet et al., 1983; Clement and Kent, 1984) has allowed an increasingly thorough testing of current transitional field models (Hoffman, 1982). These models were developed for the Matuyama-Brunhes reversal, the

\footnotetext{
* Now at Ocean Drilling Program, and Dept. Geophysics
} Texas A\&M University, College Station, Texas 77843, U.S.A. first reversal for which multiple transition records became available. The models take distinctly different approaches to how the transitional field is treated. For example, one is purely descriptive, using zonal spherical harmonics (Williams and Fuller, 1981). Another is based on a specific reversal mechanism, with the reversal beginning in a localized region of the Earth's core and flooding through the rest of the core (Hoffman, 1977). Within the framework of each model, specific field geometries may be generated which in turn predict specific, site dependent transitional records. These geometries may be tested by examining a geo- 
graphic distribution of transition records. Failure of the observed records to match those predicted may mean that the particular field geometry (i.e., harmonic content) did not exist or that the implied or explicit reversal mechanism assumed by these models is incorrect.

Evidence now exists that different reversals were characterized by different field geometries (Williams and Fuller, 1982). This further complicates the testing of transitional field models because it may not be appropriate to compare transition records from different reversals. Therefore multiple records of each reversal are needed to clearly define the field geometries. The differences between transitional fields may be more readily constrained by examining sequential reversals from the same site, thereby removing the site dependence uncertainty.

Sequential transition records have been obtained from Crete (Valet and Laj, 1981; Valet et al., 1983) and Kauai (Bogue and Coe, 1982). The results from Crete support a flooding model in which the reversal is initiated in the same region of the core for each reversal regardless of the sense of the reversal ( $R$ to $N$ or $N$ to $R$ ). The Kauai transitions, however, argue for a standing field which persisted through both reversals (Bogue and Coe, 1982). The only way to reconcile the flooding model with the Kauai data is if the region in which the reversal is initiated is dependent upon the sense of the reversal. Bogue and Coe (1982) argued that this is unlikely given the lack of evidence for a polarity asymmetry in the time averaged paleomagnetic field (Merrill and McElhinny, 1977; Merrill et al., 1979).

Examination of other sets of sequential transitions is necessary to further evaluate the existence of persistent transitional fields. The site dependence inherent in models of transitional fields requires observations from geographically distributed sites to adequately constrain the variables involved. In particular, Southern Hemisphere records are critical for establishing the extent of symmetries about the equator. The three transitions reported by Opdyke et al. (1973) from deepsea core RC14-14 were ideal candidates for further study because they represented three sequential reversals from the Southern Hemisphere.
The magnetostratigraphy of core RC14-14 $\left(35.91^{\circ} \mathrm{S}, 59.97^{\circ} \mathrm{E}\right)$ presented by Opdyke et al. (1973) indicated that the core consists of predominantly reversely magnetized sediment with two normal polarity zones occurring from about 450 to $930 \mathrm{~cm}$ and from $2250 \mathrm{~cm}$ to the bottom of the core $(2600 \mathrm{~cm})$. The two normal zones were correlated with the Jaramillo and Olduvai subchrons based on biostratigraphic control. The excellent quality of the magnetic data from this core encouraged us to resample the three transition zones using a detailed sampling technique described elsewhere (Kawai et al., 1976; Clement et al., 1982). Results from the lower Jaramillo transition ( $R$ to $N$ ) have been reported previously (Clement and Kent, 1984) and the results from the upper Jaramillo ( $N$ to $R$ ) and upper Olduvai ( $N$ to $R$ ) reversals are discussed here.

\section{Upper Jaramillo}

The original measurements made by Opdyke et al. (1973) indicated that the record of the upper Jaramillo transition contained less detail than the other two transitions in this core. This interval was resampled from 435 to $490 \mathrm{~cm}$ by taking successive $0.5 \mathrm{~cm}$ thick samples which were then subdivided to give three specimens at each sampling level. Sampling on this scale was successful in increasing the detail of the record, but several features of this record cause the interpretation of these results as an accurate record of transitional field behavior to be placed in question.

Progressive A.F. demagnetization of specimens from the full reverse and normal polarity zones is successful in removing the less stable components and identifying characteristic magnetizations (ChRM) (Zijderveld, 1967) (Fig. 1a,b). However, progressive A.F. demagnetization of specimens from within the transition zone revealed less straightforward behavior. The results typical of intermediate specimens (Fig. 1c) indicate that in some cases linear trajectories may be defined, but in the majority of the cases these did not decay towards the origin. Such behavior suggests that two or more components may be present which cannot be properly separated using A.F. demagne- 
A
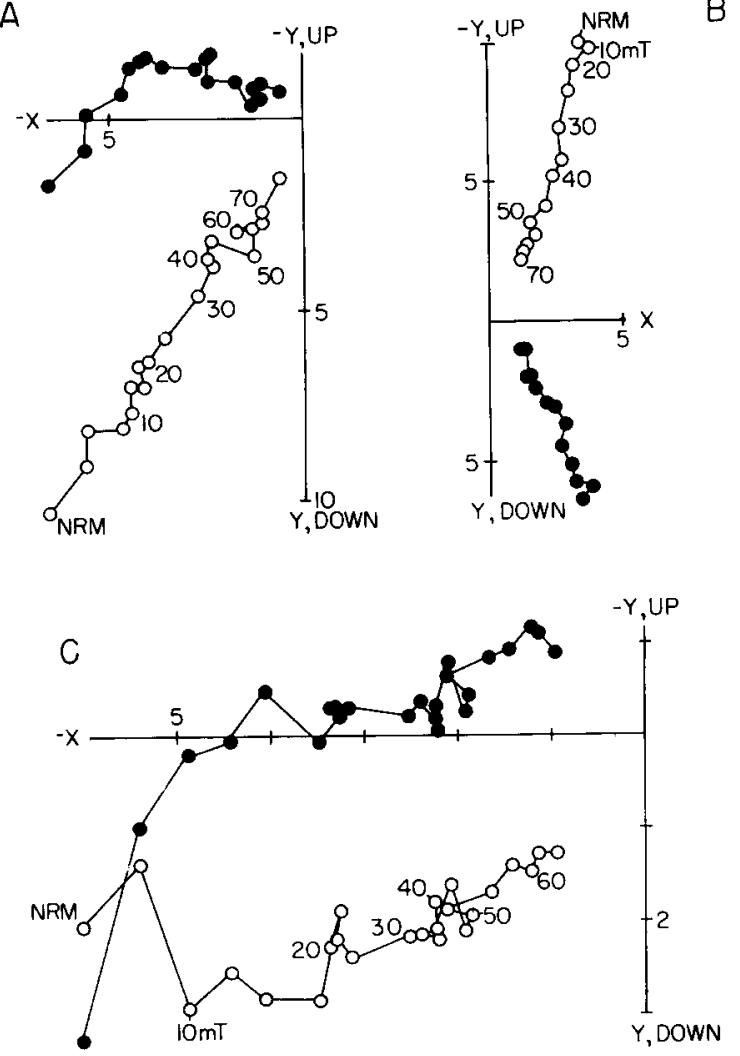

Fig. 1. Vector endpoint diagrams illustrating the results of progressive A.F. demagnetization of specimens from the upper Jaramillo polarity transition. Specimens A and B are from the reversed polarity above the reversal and the normal polarity zone below it, respectively. Specimen $\mathrm{C}$ is from within the transition zone itself. Open symbols are projections onto the vertical plane and solid symbols are projections onto the horizontal plane. Units are in $10^{-6} \mathrm{Am}^{2} \mathrm{~kg}^{-1}$.

tization. Progressive thermal demagnetization of intermediate specimens also yielded similar results which suggest that unresolvable multiple components are present. The inability of both A.F. and thermal demagnetization to resolve these components raises the question of the nature of these magnetizations. Whatever the specific reason for this behavior may be, whether related to a more complex acquisition process or rock magnetic properties of the sediment, it is clear that the details of this polarity transition are obscured.

A peak demagnetizing field of $15.0 \mathrm{mT}$, which succeeded in isolating ChRM's in the full polarity zones above and below the transition, was applied to the remaining specimens from this sampling interval in an attempt to characterize the general features of this reversal (Fig. 2). The upper Jaramillo reversal is identified by the transition from normal polarity directions below $468 \mathrm{~cm}$ to reverse polarity directions above $453 \mathrm{~cm}$. Mean inclinations of $-59.2^{\circ}$ and $54.9^{\circ}$ from the normal and reverse polarity zones, respectively, are in good agreement with predicted dipole values of $55^{\circ}$ for this site latitude. The declination record exhibits a full $180^{\circ}$ change, although it is complicated by a gradual decreasing trend away from values antipodal to normal polarity ones above $450 \mathrm{~cm}$.

The transition record is further complicated by the presence of sharp breaks in the directional as well as the intensity data. In particular the boundaries of the transition zone are very abrupt. At $468 \mathrm{~cm}$ an offset of $160^{\circ}$ is observed in the declination record. At this same level large offsets are also seen in the inclination and intensity records and again at $453 \mathrm{~cm}$, discontinuities occur in all three records. Such behavior would not be expected if the record had been smoothed as a result of a thick lock-in zone which allowed different magnetic grains from the same stratigraphic level to record the geomagnetic field at different times (Lovlie, 1976; Tucker, 1980; Otofuji and Sasjima, 1981; Denham and Chave, 1982).

The transition zone, distinctly bounded by these discontinuities, is $15 \mathrm{~cm}$ thick. A duration of 2200 $\mathrm{y}$ is calculated for this reversal using the sedimentation rate of $6.7 \mathrm{~cm} 10^{-3} \mathrm{y}$ based on the thickness of the Jaramillo Subchronozone (Clement and Kent, 1984) and the duration of the Jaramillo Chron (Mankinen et al., 1980). Compared with the durations calculated from the other two transitions in this core, the lower Jaramillo duration appears very short. This suggests that either this reversal occurred very quickly, or that the actual sedimentation rate across this interval was lower than that calculated using the thickness of the Jaramillo Subchronozone. This latter interpretation is consistent with the presence of hiatuses in this section. 

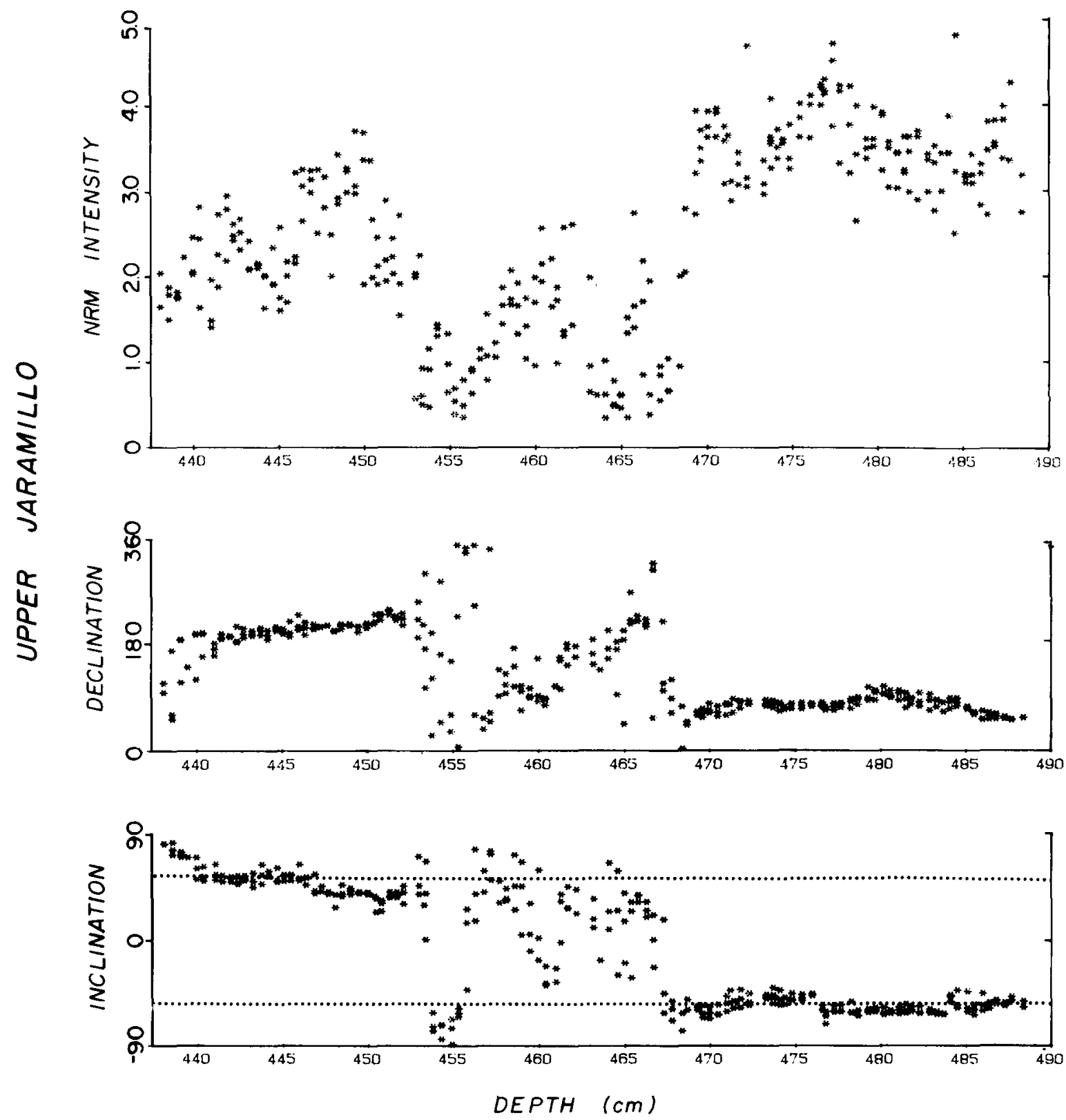

Fig. 2. Inclination, declination, and NRM intensity (normalized by specimen weight) after treatment at $15 \mathrm{mT}$ plotted versus sub-bottom depth across the upper Jaramillo reversal. The dotted lines on the inclination plot indicated the predicted values for the site latitude by normal and reversed axial dipole fields. The intensities are plotted in units of $10^{-5} \mathrm{Am}^{2} \mathrm{~kg}^{-1}$.

\section{Upper Olduvai}

Opdyke et al.'s (1973) record of the upper Olduvai transition was slightly more detailed than that of the upper Jaramillo. Resampling this inter- val, from 2220 to $2280 \mathrm{~cm}$, produced a record with considerably more detail. This record contains significantly less scatter than the upper Jaramillo and exhibits a steady progression of intermediate directions through the reversal. For these and other 
A

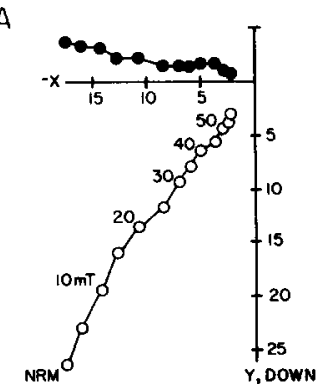

C

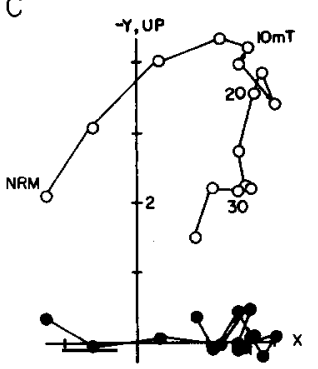

B

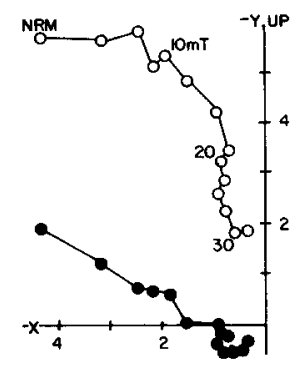

D

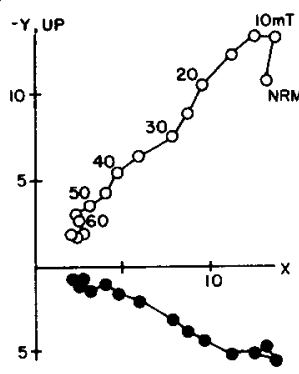

Fig. 3. Vector endpoint diagrams showing behavior of specimens from the upper Olduvai transition during progressive A.F. demagnetization. Specimens $A$ and $D$ are from the reversed (above) and normal (below) polarity zones, while specimens $B$ and $C$ are from within the interval yielding intermediate directions. Units are $10^{-6} \mathrm{Am}^{2} \mathrm{~kg}^{-1}$. See caption to Fig. 1 for further explanation.

reasons discussed below, these results may be more readily interpreted as a record of the transition from normal to reverse polarity.

The results of progressive A.F. demagnetization of four representative specimens from the Olduvai are plotted as vector endpoint diagrams in Fig. 3. In specimens from both the reverse and normal polarity zones it is clear that by treatment at 10 $\mathrm{mT}$, stable, univectorial components have been isolated (Fig. 3a,d). Specimens from within the transition zone exhibit more complex demagnetization behavior (Fig. 3b,c) although it appears that a final univectorial component can be isolated after treatment at $17.5 \mathrm{mT}$.

Detailed progressive A.F. demagnetization on specimens from below the reversal and within the transition zone revealed a consistent component

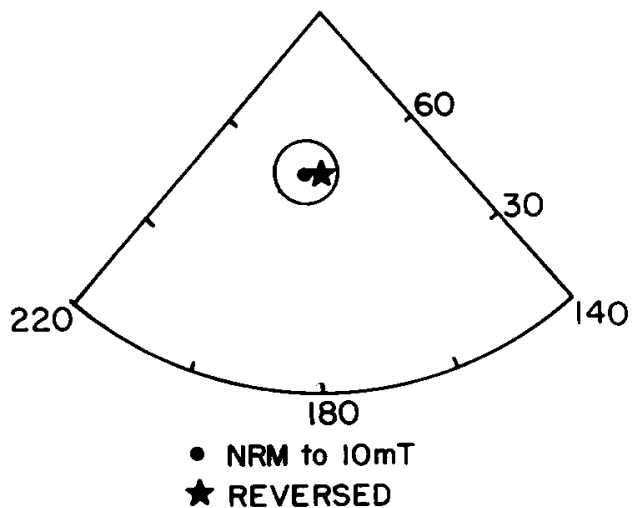

Fig. 4. The mean direction of the low coercivity component $\left(\right.$ decl $=186.6^{\circ}$, incl $\left.=54.4^{\circ}, k=30.1, \alpha_{95}=9.0^{\circ}\right)$ observed in eight specimens from the normal polarity and transition zones from the upper Olduvai section, plotted with its $95 \%$ cone of confidence on an equal area stereographic projection. Also plotted is the mean of the reversed polarity zone above the reversal.

parallel to the reverse polarity direction, which is removed by treatment at $10-15 \mathrm{mT}$. The mean direction for this low coercivity component, obtained by principal component analyses (Kirshvink, 1980) on eight specimen demagnetizations, is statistically indistinguishable from the reverse polarity direction observed upcore (Fig. 4). This low coercivity component is approximately the same magnitude across the interval while the high coercivity component decreases in strength. Therefore within the zone of low intensities the low coercivity component becomes a larger percentage of the NRM as is evident in Fig. 3c,d.

The transition record obtained after treatment at $17.5 \mathrm{mT}$ is shown in Fig. 5. The reversal from normal (mean: $\operatorname{decl}=16^{\circ}$, incl $=-50.1^{\circ}, k=$ 87.1, $\alpha_{95}=2.3^{\circ}$ for $n=44$ levels) to reverse (mean: $\operatorname{decl}=184.7^{\circ}$, incl $=54.1^{\circ}, k=220.2, \quad \alpha_{95}=1.4^{\circ}$ for $n=44$ levels) polarity directions is identified by a gradual $180^{\circ}$ shift in directions which is accompanied by a marked intensity low. Proceeding upcore from the base of the sampled interval, the NRM intensities start to decrease before the directions begin to significantly vary from the normal polarity direction. While the intensities are still dropping, the inclinations exhibit a shallowing from $-50^{\circ}$ to $-30^{\circ}$. The inclinations then steepen to nearly vertical upward directions $\left(-80^{\circ}\right)$ 

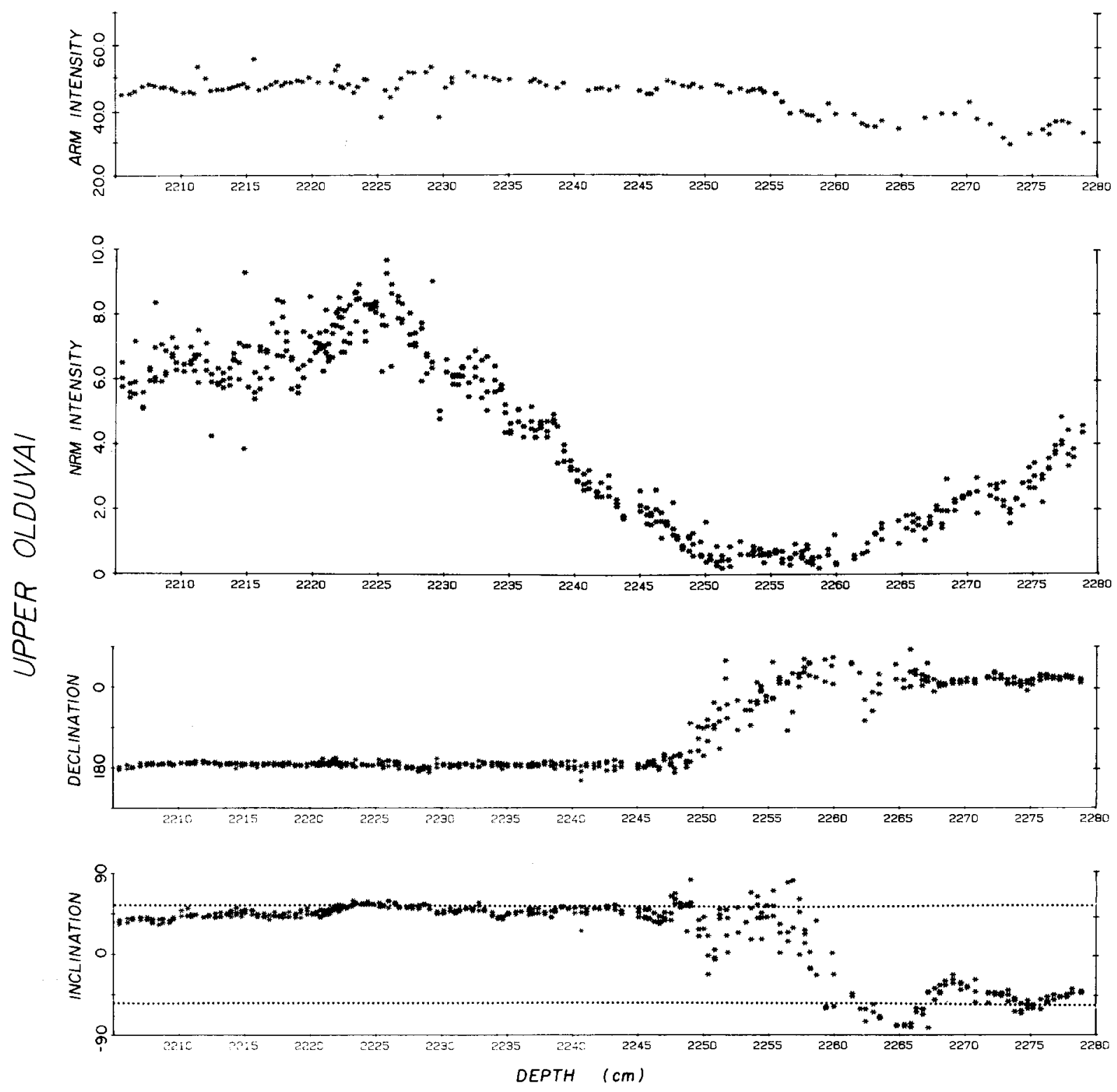

Fig. 5. The transition record obtained across the upper Olduvai after treatment at $17.5 \mathrm{mT}$. Inclination, declination, NRM and ARM intensities are plotted with sub-bottom depth. Both the NRM and ARM intensities are normalized by specimen weight and are plotted in units of $10^{-5} \mathrm{Am}^{2} \mathrm{~kg}^{-1}$. Axial dipole inclinations for normal and reverse polarity are indicated on the inclination plot by dotted lines.

and then shallow again, this time passing through the horizontal and finally settling about the reverse polarity mean. The intensities reach a minimum at approximately the same level that the directions pass through the horizontal. Meanwhile the declinations show no variation until the inclinations have closely approached reverse polarity values. A gradual progression through $180^{\circ}$ is then 
observed in the declination record until by 2247 $\mathrm{cm}$ the polarity reversal is completed. The intensities remain low until the directional change has been virtually completed and then begin an increasing trend which continues for $25 \mathrm{~cm}$ upcore.

An increase in the scatter is observed in the inclination record within the transition zone although the quality of the serial relationship through this interval remains good. The increase in the scatter in the declination record is associated with very steep inclination values and is not indicative of the scatter of the total vector.

The change in NRM intensity observed across this transition may reflect a change in the nature or amount of magnetic carrier in the sediment, or it may be a record of relative geomagnetic field intensity (Irving and Major, 1964; Kent, 1973; Barton et al., 1980). ARM measurements were used to monitor changes in the magnetic carrier (Levi and Banerjee, 1976) on the scale of this study. Specimens from the sampled interval were given an ARM using a $170 \mathrm{mT}$ A.C. field coaxial with a $0.05 \mathrm{mT}$ field based on the results of ARM acquisition experiments. The ARM intensities, normalized by specimen weight are plotted versus subbottom depth in Fig. 5. Changes in ARM intensities with depth downcore are present and may indicate that variations exist in the magnetic carrier in the sediments; however, the position and magnitude of the changes are not sufficient to account for the drop in NRM intensities within the transition zone to $5 \%$ of the full polarity values. The possible contribution of magnetic grain size changes may be examined by comparing magnetic susceptibility versus ARM (Banerjee et al., 1981; King et al., 1982). The susceptibility and ARM values across this reversal are those reported by Opdyke et al. (1973). The previously reported data were used because susceptibilities could not be accurately measured on the small specimens used here. These ARM intensities show a $40 \%$ difference between the maximum and minimum values, similar to the range measured here, while the susceptibilities show a maximum difference of 42\%. When ARM and susceptibility are plotted against each other, a cluster of points is observed

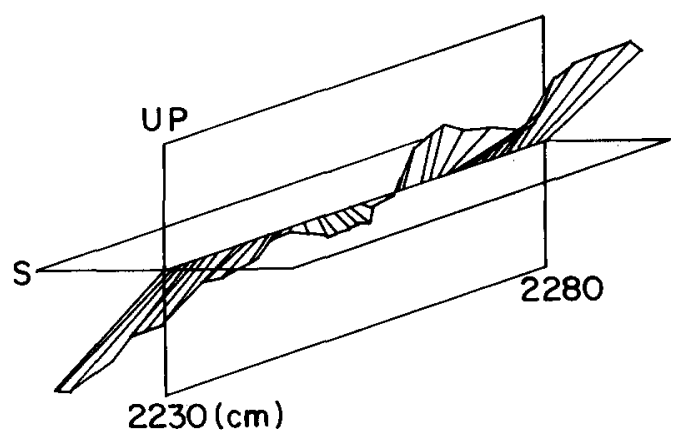

VERTICAL

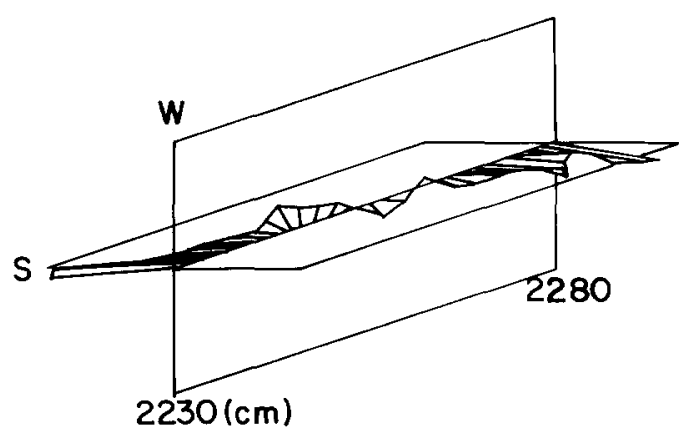

HORIZONTAL

Fig. 6. Vertical and horizontal projections of the $17.5 \mathrm{mT}$ vector endpoints plotted versus sub-bottom depth for the upper Olduvai transition. The figures are drawn looking down-core as indicated by the labeled depths. At each sample depth the vector endpoint corresponding to the projection of the measured magnetization vector onto the appropriate plane (vertical or horizontal) is plotted relative to a set of two orthogonal axes positioned so that the origin is located at the sample depth. The projected vector endpoint is then connected by a line to the sample depth so that each of these lines lies within the plane defined by the two orthogonal axes and the sampling level. In other words, the figures are the same as the more familiar two dimensional vector endpoint diagrams with the origins of the figures offset along the depth axis. To clarify the progression of the directions the projected endpoints have been connected to the endpoints corresponding to the depths immediately above and below. In this case the vector endpoints obtained after taking a three point running unit vector mean of the record after treatment at $17.5 \mathrm{mT}$ were plotted in order to simplify the diagram. The resulting figure is an effective way of combining directional and intensity data. Unfortunately the entire vector cannot be plotted on one depth axis without losing the orthogonality of the axes. The apparent shading which may occur due to the density of the data requires that the axes remain orthogonal so that the real (not apparent) directions may be read from the figure. 
with no apparent correlation with sample depth, suggesting that magnetic grain size variations are not important enough to account for the observed NRM intensity variation seen across this interval. Instead, the results of these experiments suggest that the NRM intensity pattern may provide a record of the relative change in the geomagnetic field during the deposition of these sediments.

The results across this transition from 2230 to $2278 \mathrm{~cm}$ are also shown as vector endpoint projections onto the vertical and horizontal planes plotted against depth in Fig. 6. Plotting the upper Olduvai transition in this manner illustrates the relation between the direction change and the accompanying intensity low. It should be noted that the entire record is not plotted in this figure, but only that interval encompassing the full directional change; the intensity keeps increasing up core but this interval was not included so that the details of the directional change would be more strongly emphasized. Moving upcore, the vertical component shallows and then steepens corresponding to the same trends seen in the inclination record early in the reversal. After passing through the vertical the intensity drops off dramatically as the horizontal component exhibits a $180^{\circ}$ change. The intensities then begin to rise rapidly after the directions have finished changing.

\subsection{VGP path}

The paleomagnetic directions across the upper Olduvai transition were placed in a geographic coordinate system by uniformly reorienting the declination record so that the mean normal and reverse polarity values reached the best fit about the meridian. VGP's were calculated from the unit vector mean of the three specimen directions observed at each sampling level. The resulting VGP path is plotted in Fig. 7. In the early portion of the reversal, the VGP's move along a great circle that passes through the antipode of the core site location. This behavior corresponds to that portion of the record in which the inclinations shallow and then steepen to near vertical, before the declinations begin to change. The VGP's then begin to spiral in a westward sense to more southerly latitudes and eventually cluster about the south

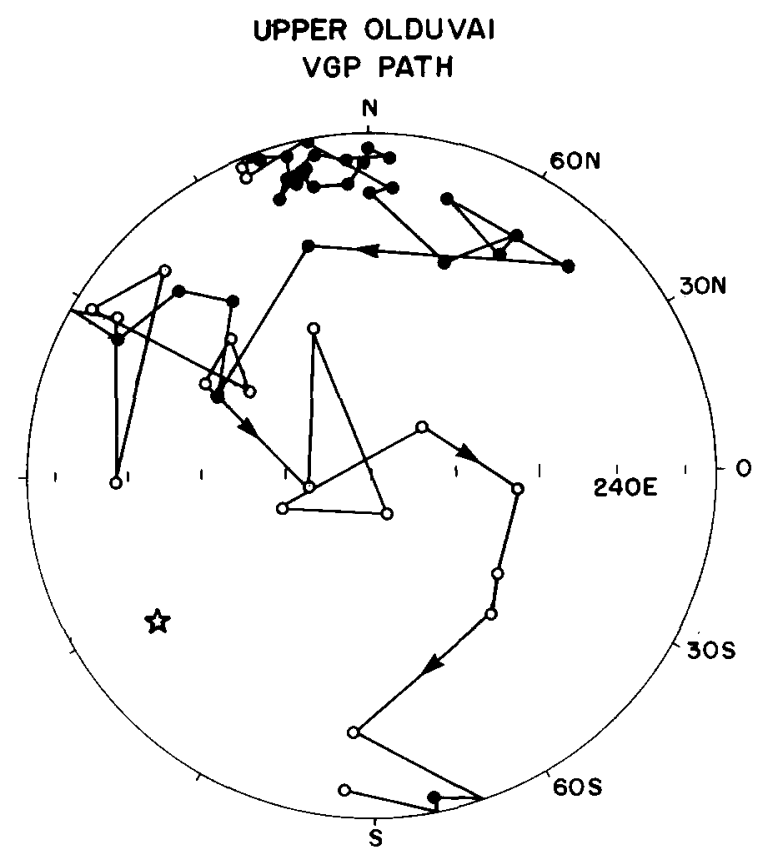

Fig. 7. Virtual geomagnetic pole path for the upper Olduvai (N to R) polarity transition. Solid (open) symbols are plotted on the near (far) hemisphere centered about the $180^{\circ}\left(0^{\circ}\right)$ meridian Each VGP was calculated using the unit vector mean of the three directions observed at each sampling level, after uniform adjustment of declinations (see text)

geographic pole, completing the normal to reverse transition. The westward sense of movement of the VGP's may correspond to a westward drift of the nondipole field during the reversal, although this could also be a combined effect of the growth and decay of nearby features of the non-dipole field without the features necessarily having to drift.

\subsection{Duration}

The duration of the upper Olduvai reversal may be estimated using the thickness of the observed transition zone and the sedimentation rate determined for this core. A problem exists, however, in that the magnetostratigraphy of this core as determined by Opdyke et al. (1973) indicated that the sedimentation rate was not constant over the entire core. In particular, the Jaramillo Subhronozone is too thick compared to the thickness of the reverse polarity zone between the top of the Olduvai Subchronozone and the base of the 
Jaramillo Subchronozone. The matter is further complicated in that the core did not penetrate the base of the Olduvai Subchronozone.

The sedimentation rate determined using the Jaramillo boundaries of 930 and $460 \mathrm{~cm}$ and the duration of the Jaramillo Subchron of $70000 \mathrm{y}$ (Mankinen et al., 1980) is $6.7 \mathrm{~cm} 10^{-3} \mathrm{y}$. Using the interval between the base of the Jaramillo (930 $\mathrm{cm})$ and the top of the Olduvai $(2260 \mathrm{~cm})$ and the duration of $680000 \mathrm{y}$ a much reduced sedimentation rate of $1.95 \mathrm{~cm} 10^{-3} \mathrm{y}$ is obtained. Using the top of the Olduvai and the base of the core gives a minimum estimate of the thickness of the Olduvai Subchronozone of $328 \mathrm{~cm}$, which together with a duration of $200000 \mathrm{y}$ (Berggren et al., 1985) yields a minimum sedimentation rate estimate of $1.6 \mathrm{~cm}$ $10^{-3} \mathrm{y}$.

The boundaries of the directional transition zone are defined as the levels at which the observed directions begin to systematically exceed the circular standard deviation of the unit vector means of the normal (reverse) polarity zone below (above) the reversal ( 6 and $9^{\circ}$, respectively). The transition zone boundaries are placed at 2270.2 and 2247.1 $\mathrm{cm}$ by this definition, resulting in a total thickness of $23.1 \mathrm{~cm}$.

The range of duration estimates of the upper Olauvai reversal calculated using the different sedimentation rate estimates is from 3500 to 14100 y. Given the variation in sedimentation rates seen in this core, the most local estimate of sedimentation rate would be expected to give the most accurate duration estimate. Since the base of the Olduvai Subchronozone was not penetrated by the core the duration estimate based on the thickness of the Olduvai is likely to be a maximum, and the true duration is probably $<14000 \mathrm{y}$. Therefore the rate based on the reverse polarity interval between the Jaramillo Subchronozone and the top of the Olduvai Subchronozone is the appropriate rate to use; it gives a duration estimate of $11100 \mathrm{y}$.

Because it was not possible to sample downcore below $2279 \mathrm{~cm}$, the full thickness of the intensity variation associated with the directional change cannot be determined. Therefore only a minimum estimate of its duration can be made. If the intensity high which extends from 2230 to $2217 \mathrm{~cm}$ is considered as transitional behavior then the inten- sity variation is observed across $62 \mathrm{~cm}$ of section. Using the calculated sedimentation rates of 6.7-1.6 $\mathrm{cm} 10^{-3} \mathrm{y}$, duration estimates of $9.3 \times 10^{3}$ to $38.7 \times 10^{3} \mathrm{y}$, respectively, are obtained.

\section{Discussion}

The record of the upper Jaramillo reversal appears to be complicated by unresolvable multicomponent magnetizations and the possible presence of hiatuses in this part of the section. This behavior contrasts with results from the lower Jaramillo and upper Olduvai transitions which represent more straightforward records. As discussed previously, progressive A.F. demagnetization of even transitional specimens from the upper Olduvai reversal was successful in removing a consistent, low coercivity overprint, isolating a characteristic remanent magnetization in these specimens. Because this overprint is statistically identical to the stable reverse polarity direction recorded above the transition, it is difficult to ascribe its origin to a magnetization acquired either during storage or in situ during the Bruhnes Chron (normal polarity). While this component may have been acquired in situ, the sediments have been exposed to predominantly normal polarity for the past $0.73 \mathrm{Ma}$ (Bruhnes), during which a reverse polarity viscous component acquired during the preceeding Matuyama Chron would be likely to have been removed or greatly reduced.

The low coercivity component also cannot be readily explained in terms of a theory in which the smallest magnetic grains (expected to have the higher coercivities) are more likely to be physically reoriented within the sediment by a subsequent field (Tucker, 1980; Denham and Chave, 1982). Opdyke et al. (1973) demonstrated that the principal magnetic material in this core is magnetite and titanomagnetite. Therefore the largest grains would have on the average the lowest coercivities and would be expected to have the lowest grain mobility. Although the origin of this component remains uncertain, the results illustrated in Fig. 3 indicate that A.F. demagnetization can successfully remove it, leaving a stable univectorial magnetization interpretable as a record of field behavior. 

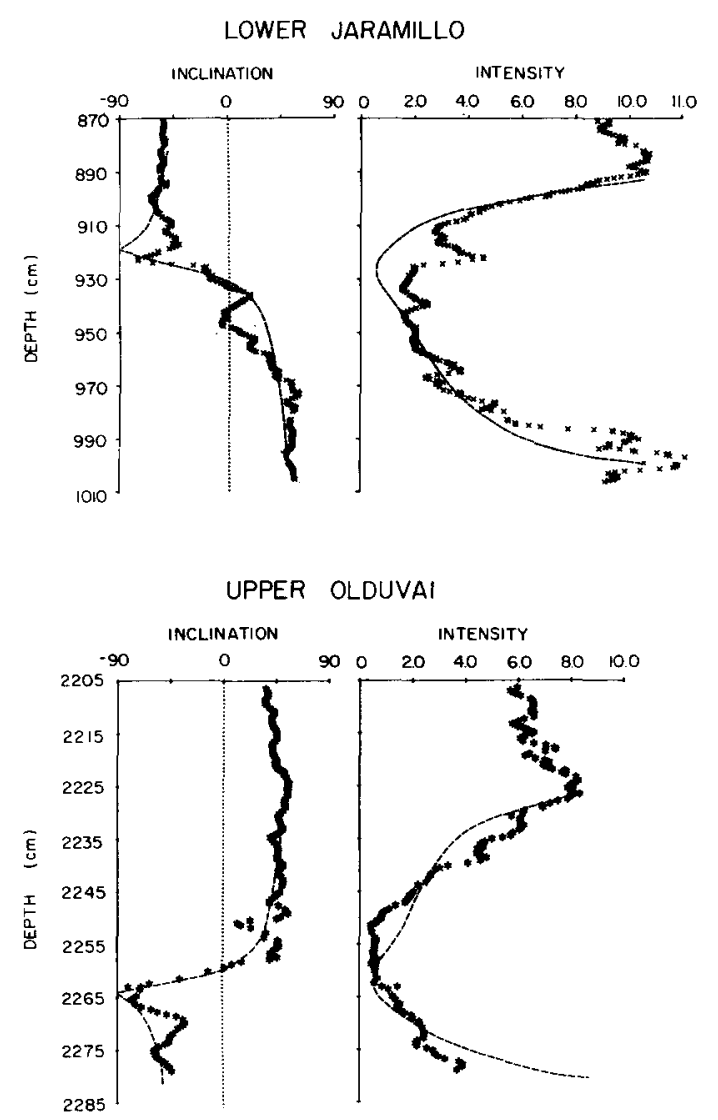

Fig. 8. The inclination and intensity records obtained across the upper Olduvai and lower Jaramillo polarity transition plotted versus sub-bottom depth. The data were smoothed using a three and five point running unit vector mean, respectively. The intensities are plotted in units of $10^{-5} \mathrm{Am}^{2}$. The dashed lines are the synthetic records produced using a zonal harmonic model of the transitional fields as discussed in the text.

A comparison of the results of the upper Olduvai ( $\mathrm{N}$ to $\mathrm{R}$ ) transition with those previously obtained for the lower Jaramillo transition (Clement and Kent, 1984) reveals a number of striking similarities in the directional and intensity profiles (Fig. 8).

Both transitions exhibit inclinations that pass through very steep upward values, $>-80^{\circ}$. In the upper Olduvai transition ( $\mathrm{N}$ to $\mathrm{R}$ ) this occurs early in the reversal, or before the directions pass through the horizontal, while in the $\mathrm{R}$ to $\mathrm{N}$ record (lower Jaramillo), very steep upward directions are observed late in the reversal (after passing through the horizontal). In addition to these steep negative values, both inclination records also exhibit a marked shallowing (up to $25^{\circ}$ shallower than axial dipole field directions) that occurs between the very steep directions and the full normal polarity values. While the declination records from these two reversals are significantly different, in each case the declinations do not begin to change until the inclinations have passed through the vertical. In short, the time progression of changes in the inclinations in the $\mathbf{N}$ to $\mathrm{R}$ reversal is almost exactly opposite to the progression observed in the $\mathrm{R}$ to $\mathrm{N}$ reversal.

The intensity records also exhibit notable similarities in that in each case a large decrease in NRM intensities to $<10 \%$ of the highest values observed across the sampled interval is associated with the directional change. Unfortunately the upper Olduvai record could not be extended farther downcore, and therefore the intensity behavior beneath $2279 \mathrm{~cm}$ cannot be determined. Although the lower end is unbounded in this case, the intensity lows for both the lower Jaramillo and upper Olduvai transitions extend across longer intervals than. the directional changes. Another point of similarity is that intensity highs bracket the low in the lower Jaramillo record which appear similar to the intensity high which occurs above the low in the upper Olduvai record. Corresponding highs are not reflected in the ARM measurements, suggesting that these are not simply a result of changes in the magnetic carrier. It is also difficult to attribute the intensity high to mixed magnetization components as these highs occur over intervals of both full normal and reverse polarities.

The existence of these and other similarities in these two transition records makes it difficult to discount these records as simply products of multicomponent magnetizations caused by smoothing as the sediment passes through a thick lock-in zone. Instead, the high degree of internal consistency and antisymmetry between the upper Olduvai and lower Jaramillo transition records supports our contention that they represent field behavior during these reversals. It is therefore worthwhile comparing these records with those predicted for this site latitude by some transitional field models. Although the VGP paths of these 
transitions suggest the presence of non-axisymmetric fields during these reversals, we examine the extent to which other features of these polarity transition records can be modeled using the simplified assumption of axisymmetric fields.

The zonal harmonic field model developed by Williams and Fuller (1981) is capable of producing synthetic inclination and intensity records of a polarity reversal for a site latitude. The dipole term is allowed to decay exponentially through zero and then build up again in the opposite direction. The energy lost by the dipole is distributed amongst the low order, non-dipole zonal terms. By fitting synthetic records produced in this manner to the available records of the Matuyama-Brunhes transition, Williams and Fuller (1981) were able to model this transition with 20,30 and $50 \%$ of the dipole energy going to a positive $G_{2}^{0}$, a negative $G_{3}^{0}$ and a positive $G_{4}^{0}$. As previously discussed (Clement and Kent, 1984), the record predicted for $35^{\circ}$ south latitude using these parameters in their model does not agree with the observed record of the lower Jaramillo transition. It was possible, however, to produce a fitting synthetic record by changing the energy partitioning and putting the majority of the energy lost by the dipole field into a negative quadrupole $\left(g_{2}^{0}\right)$ term.

As noted above, a feature common to both the lower Jaramillo and the upper Olduvai is that the inclinations pass through very steep negative values. An important difference, however, is that this occurs early in the $\mathbf{N}$ to $\mathbf{R}$ reversal (Olduvai) and late in the $\mathrm{R}$ to $\mathrm{N}$ reversal (Jaramillo). These were the features which controlled our modeling efforts. It appears that at this latitude $\left(35.9^{\circ}\right.$ south, which is close to the $G_{2}^{0}$ node), the model will predict inclination records which pass through negative vertical values by changing the sign of $G_{2}^{0}$ from positive to negative and putting more than $50 \%$ of the available energy into it. To shift the vertical directions toward the mid-point of the reversal, however, the energy distributed to the $G_{3}^{0}$ and $G_{4}^{0}$ terms had to be severely reduced. On the other hand, we found that by also switching the sign of the $G_{4}^{0}$ from positive to negative the observed inclination records could be modeled by maintaining the energy partitioning used by Williams and
Fuller (1981) to model the Matuyama-Brunhes reversal $\left(20,30\right.$ and $50 \%$ to $\left.G_{2}^{0}, G_{3}^{0}, G_{4}^{0}\right)$.

As shown in Fig. 8, this modified model is successful at predicting the steep negative inclinations observed in these records. The position of the steep inclinations within the transition is also accounted for even though the models were pinned according to the intensity records. The scaling of the modeled records was determined by pinning the beginning and end of the synthetic record to the beginning and end, respectively, of the intensity low in the lower Jaramillo. In the upper Olduvai, the end of the modeled record was pinned to the end of the intensity low. The initial intensity magnitudes of the synthetic records were scaled to the observed intensity highs. The same model parameters are used to model the lower Jaramillo and the upper Olduvai. The only differences in the model used here for the upper Olduvai and the lower Jaramillo, and that of Williams and Fuller (1981) for the Matuyama/Brunhes reversal, is that the signs of the two zonal terms $\left(G_{2}^{0}, G_{4}^{0}\right)$ which are symmetric about the equator have been reversed.

The purpose of this modeling exercise was not to try to determine a very exact harmonic content for each of these reversals. There are a number of combinations of variables in this type of model which may produce very reasonable fits. Until more geographical control is obtained, it also does not seem warranted to attempt more complicated variants, for example, including non-zonal terms. Nevertheless the general success of the model as used suggests that very similar transitional fields may have been present during both the upper Olduvai and lower Jaramillo reversals and that the differences in relative timing of many of the features of these sequential transition records are mostly a function of the different sense of the reversals.

The modeling results are consistent with the idea of a standing field which persisted across both of these reversals. Although the Williams-Fuller model does not treat the non-dipole terms strictly as a standing field (their absolute magnitudes vary), their relative proportions and signs do remain constant as the dipole term reverses. When the same such standing field is 
maintained across a normal to reverse and a reverse to normal reversal, the resulting transition records appear to be antisymmetric in that the same features are observed in the opposite order. This is precisely what is observed in these two Southern Hemisphere records.

The two Southern Hemisphere records discussed here therefore agree with the sequential transitions obtained from Kauai (Bogue and Coe, 1982) in providing evidence for persistent standing fields. These results differ from the sequential Miocene records from Crete (Valet and Laj, 1981; Valet et al., 1983) which support the dependence of the near or far sided nature of the transitional VGP path on the sense of the reversal ( $R$ to $N$ or $\mathrm{N}$ to $\mathrm{R}$ ). Comparing the inclination and intensity records from two of the Crete records (Valet and Laj, 1981), however, reveals that although the reverse to normal VGP path is predominately nearsided, the inclinations pass through very steep negative values early in the reversal; the normal to reverse transition exhibits steep negative inclinations late in the reversal. Therefore if only the inclination and intensity records are considered, these Crete records appear similar to the sequential transition records shown here from RC14-14, but with an opposite sense of timing of the steep inclinations. Initial modeling of the Crete records indicates that the difference in relative timing of the steep negative inclinations within the transitions may be accounted for by the difference in site locations.

In summary, there are now three sets of sequential transition records available, but the combined data set may not be consistent with a particular transitional field geometry or a particular reversal mechanism. Two of the sets, one from Kauai (Bogue and Coe, 1982) and the set discussed here from the southern Indian Ocean core, argue for the persistence of a similar transitional (i.e., standing) field across sequential reversals. The set from Crete (Valet and Laj, 1981; Valet et al., 1983) is instead more readily explained by a flooding reversal mechanism (Hoffman, 1977). If the standing field interpretation is viewed from the framework of a flooding mechanism, then it would imply that the reversal is improbably initiated in opposite hemispheres dependent upon the sense of the reversal. On the other hand, without a geographic distribution of records of the same reversal(s), the standing field hypothesis is left poorly constrained. Despite the apparent between-site discrepancies, the within-site consistency observed in each of the available sequential transition record sets leads us to believe that there are real spatial and temporal variations in fields which have yet to be explained by existing transitional field models.

\section{Acknowledgments}

We thank J.L. LaBrecque and S.C. Cande and an anonymous reviewer for helpful comments which led to improvements in the manuscript. This work was supported by the National Science Foundation, Oceanography, under grant OCE8300356. Lamont-Doherty contribution 3837.

\section{References}

Banerjee, S., King, J. and Marvin, J., 1981. A rapid method for magnetic granulometry with applications to environmental studies. Geophys. Res. Lett., 8: 333-336.

Barton, C.E., McElhinny, M.W. and Edwards, D.J., 1980. Laboratory studies of depositional DRM. Geophys. J.R. Astron. Soc., 61: 355.

Berggren, W.A., Kent, D.V. and Van Couvering, J.A., 1985. Neogene geochronology and chronostratigraphy. In: N.J. Snelling (Editor), Geochronology and the Geologic Time Scale. Geol. Soc. London Spec. Publ., in press.

Bogue, S.W. and Coe, R.S., 1982. Back to back paleomagnetic reversal records from Kauai. Nature, 295: 399-401.

Clement, B.M. and Kent, D.V., 1984. A detailed record of the lower Jaramillo polarity transition from a southern hemisphere deep-sea sediment core. J. Geophys. Res., 89: 1049-1058.

Clement, B.M., Kent, D.V. and Opdyke, N.D., 1982. Brunhes-Matuyama polarity transition in three deep-sea cores. Philos. Trans. R. Soc. London, A306: 113-119.

Denham, C.R. and Chave, A.D., 1982. Detrital remanent magnetization: viscosity theory of the lockin zone. J. Geophys. Res., 87: 7126-7130.

Hoffman, K.A., 1977. Polarity transition records and the geomagnetic dynamo. Science, 196: 1329.

Hoffman, K.A., 1982. The testing of geomagnetic reversal models: recent developments. Philos. Trans. R. Soc. London, A306: 147-154.

Irving, E. and Major, A., 1964. Post-depositional detrital remanent magnetization in a synthetic sediment. Sedimentology, 3: $135-143$. 
Kawai, N., Otofuji, Y. and Kobayoshi, K., 1976. Paleomagnetic study of deep-sea sediments using thin sections. J. Geomagn. Geoelectr., 28: 395-412.

Kent, D.V., 1973. Post depositional remanent magnetization in deep-sea sediment. Nature, 246.

King, J., Banerjee, S.K., Marvin, J. and Ozdemir, O., 1982. A comparison of different magnetic methods for determining the relative grain size of magnetite in natural materials: some results from lake sediments. Earth Planet. Sci. Lett., 59: 404-419.

Kirschvink, J.L., 1980. The least squares line and plane and the analysis of paleomagnetic data. Geophys. J.R. Astron. Soc. 62: 699-718.

Levi, S. and Bannerjee, S.K., 1976. On the possibility of obtaining relative paleointensities from lake sediments. Earth Planet. Sci. Lett., 29: 219-226.

Lovlie, R., 1976. The intensity pattern of pDRM acquired in some marine sediments deposited during a reversal of the external magnetic field. Earth Planet. Sci. Lett., 29: 209-214.

Mankinen, E.A., DonnellyNolan, J.M., Gremme, C.S. and Hearn, B.C., Jr., 1980. Paleomagnetism of the Clear Lake volcanics and new limits on the age of the Jaramillo polarity event. Geol. Sur., Prof. Pap. 1141, 6782.

Merrill, R.T. and McElhinny, M.W., 1977. Anomalies in the time-averaged paleomagnetic field and their implication for the lower mantle. Rev. Geophys. Space Phys., 15: 309-323.

Merrill, R.T., McElhinny, M.W. and Stevenson, D.J., 1979. Evidence for long term asymmetries in the Earth's magnetic field and possible implications for dynamo theories. Phys. Earth Planet. Inter., 20: 75-82.

Opdyke, N.D., Kent, D.V. and Lowrie, W., 1977. Details of magnetic polarity transitions recorded in a high deposition rate deep-sea core. Earth Planet. Sci. Lett., 20: 315-324.

Otofuji, Y. and Sasjima, S., 1981. A magnetization process of sediments: laboratory experiments on pdrm. Geophys. J.R. Astron. Soc., 66: 241-259.

Tucker, P., 1980. Stirred remanent magnetization: a laboratory analogue of post depositional realignment. Geophys. J.R. Astron. Soc., 48: 153-157.

Valet, J.P. and Laj, C., 1981. Paleomagnetic record of two successive Miocene geomagnetic reversals in western Crete. Earth Planet. Sci. Lett., 54: 53-63.

Valet, J.P., Laj, C. and Langereis, C.G., 1983. Two different R-N geomagnetic reversals with identical VGP paths recorded at the same site. Nature, 304: 330-332.

Williams, I. and Fuller, M., 1981. Zonal harmonic models of reversal transition records. J. Geophys. Res., 86: 11657-11665.

Williams, I. and Fuller, M., 1982. A Miocene polarity transition (R-N) from the Agno batholith, Luzon. J. Geophys. Res., 87: 9408-9418.

Zijderveld, J.D.A., 1967. A.C. demagnetization of rocks: analysis of results. In: D.W. Collinson, K.M. Creer, and S.K. Runcorn (Editors), Methods in Paleomagnetism. Elsevier, New York, pp. 254-286. 